\title{
O uso do método de revisão sistemática da literatura na pesquisa em logística, transportes e cadeia de suprimentos
}

\author{
Sergio Adriano Loureiro' ${ }^{1}$ Ana Paula Reis Noletto², Lilian da Silva Santos ${ }^{3}$, \\ José Benedito Silva Santos Júnior ${ }^{4}$ e Orlando Fontes Lima, Jr. ${ }^{5}$
}

\begin{abstract}
Resumo: A revisão da literatura é uma etapa chave da pesquisa em qualquer campo da ciência. Na área de logística e transportes se destaca o uso da Revisão Sistemática da Literatura (RSL). Este método apresenta um processo replicável e auditável de execução, mas não está livre do viés e da subjetividade do pesquisador e de falhas em sua execução que podem comprometer os resultados da revisão. Diante destas dificuldades, o trabalho propõe a adoção de critérios de avaliação da qualidade na condução da RSL de forma a identificar falhas e corrigi-las. Para demonstrar a aplicabilidade da proposta, três estudos de caso na área de logística e transportes são avaliados. A aplicação permitiu identificar falhas na execução da RSL e apontar formas de correção das mesmas. Ressalta-se que os critérios propostos são úteis também para a avaliação da qualidade de revisões narrativas da literatura.
\end{abstract}

Palavras-chave: revisão sistemática, logística, transportes, cadeia de suprimento.

\begin{abstract}
The literature review is a research key stage in any field of science. The use of the Systematic Literature Review stands out in logistics and transportation, submitting a repeatable and auditable implementation process. However, SLR execution steps are not free from bias and subjectivity of the researcher. In face of these difficulties, this paper proposes the adoption of quality evaluation criteria while conducting the SLR to identify failures and to correct them. To demonstrate the applicability of the proposal, three case studies in logistics and transportation are evaluated. The application identified failures in the execution of SLR and pointed ways to correct them. It is highlighted that the proposed criteria are also useful for assessing the quality of narrative literature review.
\end{abstract}

Keywords: systematic review, logistics, transportation, supply chain.

\section{INTRODUÇÃO}

A revisão da literatura é uma etapa chave da pesquisa em qualquer campo da ciência. Através de sua execução o pesquisador determina os limites do conhecimento sobre o assunto de interesse, obtendo meios de abordar novos problemas através do conhecimento já estabelecido ou explorando novas abordagens para antigos problemas. $\mathrm{Na}$ área de logística e transportes, os métodos de Revisão da Literatura Narrativa (RLN) e a Revisão Sistemática da Literatura (RSL) são os comumente usados.

Nos últimos anos, é possível identificar uma tendência de crescimento de publicações de artigos de RSL, conforme pode ser comprovado no Gráfico 1. Este gráfico apresenta

Sergio Adriano Loureiro, Universidade Estadual de Campinas, Laboratório de Aprendizagem em Logística e Transportes (loureiro@fec.unicamp.br).

${ }^{2}$ Ana Paula Reis Noletto, Universidade Estadual de Campinas, Instituto de Tecnologia de Alimentos, Centro de Tecnologia de Embalagens (ananoletto@gmail.com).

${ }^{3}$ Lilian da Silva Santos, Universidade Estadual de Campinas, Laboratório de Aprendizagem em Logística e Transportes (lilisisa@gmail.com).

${ }^{4}$ José Benedito Silva Santos Júnior, Universidade Estadual de Campinas, Laboratório de Aprendizagem em Logística e Transportes (jbened@gmail.com).

${ }^{5}$ Orlando Fontes Lima, Jr., Universidade Estadual de Campinas, Laboratório de Aprendizagem em Logística e Transportes (oflimaj@fec.unicamp.br).

Manuscrito recebido em 11/09/2015 e aprovado para publicação em 04/03/2016.

Este artigo é parte de TRANSPORTES v. 24, n. 1, 2016 ISSN: 2237-1346 (online). DOI: 10.14295/transportes.v24i1.919 a distribuição de publicações indexadas na base de dados Compedex, acessadas através do Engineering Village, relacionadas a RLN e RSL. O Compedex indexa periódicos classificados pelo Qualis da CAPES nas diversas áreas de engenharias, além de outros periódicos com relevante fator de impacto e índice h que não constam do Qualis. Grupos editoriais como Elsevier, Springer, Taylor and Francis, John Willey and Sons, Sage Publications, Science Press e IEEE possuem material bibliográfico indexado pelo Compedex.

Os dados apresentados no Gráfico 1 foram obtidos empregando os termos de busca "literature review", "narrative literature review", "systematic review" e "systematic literature review". Foram incluídos resultados onde: os termos de busca constavam do título das publicações; os trabalhos estavam classificados nas áreas de engenharias, administração e gestão, e ciência da decisão; os trabalhos eram artigos publicados em periódicos, artigos publicados em anais de congressos e eventos, e capítulos de livros. O período considerado para levantamento foi de 2006 a 2015.

No Brasil, também, é possível observar tendência similar de crescimento. No período de 2000 a julho de 2015 podem ser identificados trinta trabalhos de revisão da literatura distribuídos na Revista Transportes, no Journal of Transport Literature JTL-RELIT e nos anais dos Congressos da Associação Nacional de Pesquisa e Ensino em Transportes (Anpet). Aproximadamente 60\% destes trabalhos foram publicados nos últimos três anos.

O crescimento do emprego do método de RSL nos últimos anos pode ser explicado através de fatores como: a existência de um processo formal e replicável de execução da revisão da literatura; a capacidade do método de consolidar grande volume de informações, analisá-los e apre- 


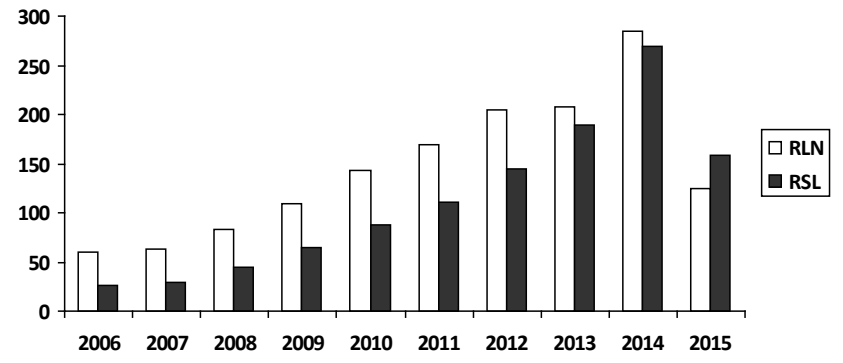

Gráfico 1: Distribuição das publicações indexadas na base de dados Compedex.

sentá-los de forma inteligível quando combinado a métodos como a Metassíntese e a Meta-análise.

Apesar desses fatores que representam vantagens do método RSL, a aplicação da RSL pode ser desvantajosa se comparada com a RLN por demandar maiores esforços de pesquisa nas etapas de identificação e seleção dos trabalhos, e por não eliminar o risco de viés que está presente na etapa de condução de avaliação da qualidade dos trabalhos (Tranfield et al. 2003, De La Torre-Ugarte-Guanilo et al., 2011; Soni e Kodali, 2011; Popay et al., 1998).

Perante essa dificuldade, pode-se adotar estratégias de condução da pesquisa como, por exemplo, a avaliação dos estudos realizada por um painel de pesquisadores. De acordo com Denyer e Tranfield (2009), o painel deve ser composto por pesquisadores com experiência e senioridade no tema de pesquisa e na condução do método de RSL.

Além do painel de pesquisadores, outras abordagens são sugeridas na literatura para reduzir o viés na condução da RSL. Popay et al. (1998), Shea et al. (2007) e Denyer et al. (2008) propõem o emprego de questões para avaliação da qualidade. Porém estas abordagens estão limitadas a etapas específicas da RSL ou ao emprego posterior à condução da RSL.

Outro aspecto a destacar é que nem sempre a correta execução do método atende aos objetivos esperados de uma revisão da literatura, já que muitas revisões podem apresentar resultados e inferências pouco relevantes para o prosseguimento da pesquisa.

Diante do exposto, este trabalho propõe o emprego de critérios de qualidade para identificação e correção das falhas à medida que as etapas da RSL são executadas. Para demonstrar a aplicabilidade da proposta, três artigos oriundos da área de logística e transportes foram avaliados mediante a aplicação do Método de Estudos de Casos Múltiplos, baseados em Yin (2005), combinados aos critérios de qualidade de RSL, adaptados de Popay et al. (1998).

As conclusões deste trabalho são resultados de pesquisas desenvolvidas por um grupo de pesquisa na área de Transportes e Logística desde 2008. O trabalho seminal do grupo nesta área foi de RSL com Metassíntese (Tarchetti, 2010) não incluso neste artigo. Desde então, outros trabalhos foram produzidos pelo grupo com sucesso empregando o método de RSL, além, é claro, de algumas outras tentativas sem muito sucesso. A partir desta experiência de aplicação do método de RSL, e comparando com o método de RLN utilizado pelo grupo desde 1999, é possível destacar:

1. O uso da RSL, embora bastante sistematizado, exige esforço e senioridade de pesquisa incompatível com a maturidade da maioria dos pesquisadores da categoria de iniciação científica e mestrado.

2. O grau de exigência e dedicação necessário para uma correta aplicação do método de RSL muitas vezes conduz o pesquisador menos experiente a confundir-se entre a valorização da aplicação do método e do objetivo da pesquisa propriamente e, na maior parte das vezes, negligenciando a etapa de conclusão.

3. A falta de familiaridade do pesquisador com o tema de pesquisa pode também penalizar a aplicação do método e os resultados obtidos, principalmente nas etapas iniciais relacionadas à: definição da questão de pesquisa; escolha dos termos de busca; e seleção das bases de dados e periódicos a serem consultados.

O artigo está organizado em seis seções, a primeira seção introduziu o tema do trabalho e seus objetivos. Na segunda seção são apresentados o processo de revisão da literatura e as etapas da RSL. Na terceira seção é discutida a necessidade da avaliação da qualidade em RSL. Na quarta é apresentado o método de pesquisa. Na quinta seção são apresentados e analisados os três estudos de caso selecionados. As conclusões do trabalho são apresentadas na sexta seção.

\section{O PROCESSO DE REVISÃO DA LITERATURA}

Artigos de revisão de literatura são úteis tanto para pesquisadores quanto para os leitores por fornecerem uma visão geral, atualizada e estruturada de uma área específica do conhecimento (Van Wee e Banister, 2015). A Tabela 1 apresenta uma classificação dos artigos de revisão da literatura considerando o valor proporcionado e os principais resultados que podem ser alcançados por este tipo de artigo.

Constata-se, pela Tabela 1, que o valor proporcionado por artigos de revisão é amplo e abrange: tornar explícitas lacunas de pesquisa; delinear vantagens e desvantagens de métodos científicos e teorias empregados a um problema; elaborar a construção de modelos conceituais; e discutir aplicações do conhecimento científico em problemas do mundo real.

De acordo com Casey e Landgraf (2015), uma revisão da literatura bem escrita e devidamente pesquisada deve proporcionar ao pesquisador e seu leitor:

- Familiarizar-se com a pesquisa: a revisão da literatura deve auxiliar o pesquisador a entender o que se sabe e o que não se sabe sobre o tópico de pesquisa, identificando áreas de controvérsia e questões que requerem mais estudos. Abordagens e métodos aplicados sem sucesso ao tópico de pesquisa também podem ser identificados e evitados.

- Fornecer contexto: a revisão da literatura deve resumir o estado atual da pesquisa para o leitor e fornecer uma visão geral dos trabalhos anteriores sobre tópicos relacionados. O significado e a relevância dos trabalhos anteriores para o presente estudo devem ser claros, as alegações apresentadas devem ser analisadas de forma crítica e as considerações formuladas através 
Tabela 1: Valor Adicionado pela revisão da literatura.

Fonte: Van Wee e Banister (2015).

\begin{tabular}{|c|c|c|}
\hline Valor Adicionado & Comentários & Resultado Principal (exemplos) \\
\hline Percepções empíricas & Uma síntese do que já é conhecido & $\begin{array}{l}\text { Estágio de conhecimento } \\
\text { Lacunas na literatura } \\
\text { Fraqueza das metodologias usadas }\end{array}$ \\
\hline Metodologias & $\begin{array}{l}\text { Uma análise dos métodos utilizados, suas vantagens e } \\
\text { desvantagens }\end{array}$ & $\begin{array}{l}\text { Visão geral de metodologias dominantes } \\
\text { utilizadas } \\
\text { Prós e contras de metodologias usadas } \\
\text { Oportunidades para novos métodos }\end{array}$ \\
\hline Teorias & $\begin{array}{l}\text { Uma investigação de diferentes teorias utilizadas, e sua } \\
\text { importância. A investigação deve incluir as implicações } \\
\text { da teoria para os resultados. }\end{array}$ & $\begin{array}{l}\text { Visão geral das principais teorias utilizadas } \\
\text { Forças e fraquezas das teorias usadas } \\
\text { Potencial de uso de outras teorias }\end{array}$ \\
\hline $\begin{array}{l}\text { Lacunas na literatura } \\
\text { e agenda de pesquisa }\end{array}$ & $\begin{array}{l}\text { Relaciona-se aos comentários feitos para revisões } \\
\text { com foco empírico, metodológico e teórico. Explorar } \\
\text { omissões e limitações nas abordagens e sugerir formas } \\
\text { para avançar o conhecimento. }\end{array}$ & $\begin{array}{l}\text { Principais lacunas na literatura } \\
\text { Oportunidade para pesquisas futuras }\end{array}$ \\
\hline $\begin{array}{l}\text { Relevância para } \\
\text { aplicações do mundo } \\
\text { real }\end{array}$ & $\begin{array}{l}\text { Uma discussão ou síntese da utilidade da literatura para } \\
\text { aplicações do mundo real (politicas, planejamento etc.) } \\
\text { - talvez com utilização de estudo de caso }\end{array}$ & $\begin{array}{l}\text { Visão geral do conhecimento disponível para } \\
\text { aplicações do mundo real; } \\
\text { Orientações de projeto; } \\
\text { Exemplos de casos reais que estão (ou não) } \\
\text { apoiados por resultados da literatura; } \\
\text { Comparação entre casos ou países; }\end{array}$ \\
\hline Modelo conceitual & $\begin{array}{l}\text { Fornecer uma estrutura explicita dos relacionamentos } \\
\text { entre variáveis dependentes e independentes. Pode ser } \\
\text { apresentada antes ou após a seção de revisão do artigo. }\end{array}$ & $\begin{array}{l}\text { Esquema, figura que apresenta o modelo } \\
\text { conceitual; } \\
\text { Visão geral de quais partes são (ou não) } \\
\text { procedentes ou apoiadas pela literatura. }\end{array}$ \\
\hline
\end{tabular}

destas considerações devem sintetizar a literatura e apresentar uma nova perspectiva.

- Estabelecer autoridade: uma revisão da literatura bem-feita demonstra a familiaridade do autor com o estado atual do conhecimento e da pesquisa em um determinado campo.

Entre os métodos de revisão da literatura que se destacam na área de Logística e Transportes estão a Revisão da Literatura Narrativa (RLN) e a Revisão Sistemática da Literatura (RSL). Na sequência são apresentadas descrições dos dois métodos, sendo que a descrição da RSL é realizada com maiores detalhes.

A RLN é o método de revisão mais usual nas ciências. O processo de desenvolvimento consiste na identificação, localização, compilação, análise e interpretação do conhecimento de fontes diversas como livros, artigos, relatórios, dissertações e teses onde os diferentes argumentos obtidos são apresentados pelo pesquisador em uma estrutura narrativa (Marconi e Lakatos, 2010). Casey e Landgraf (2015) afirmam que existem várias formas de organização e apresentação dos resultados de uma RLN:

- Cronológica: útil para demonstrar como o conhecimento em um determinado campo evoluiu e as mudanças que ocorreram.

- Descritiva: onde são apresentados os autores que escreveram sobre determinado tema, seguido da análise deste tema. Esta forma evidencia os tópicos que compõem a totalidade do tema pesquisado.

- Descritiva analítica: é uma variação da forma descritiva. Nesta forma, a análise deve contemplar as semelhanças e diferenças entre as fontes de dados disponíveis em cada tópico, ao invés de apresentar análise apenas na etapa final.

- Do maior para o menor e de volta para o maior: nesta forma de organização se inicia apresentando estudos mais gerais e amplos sobre determinado tópico, antes de se apresentarem estudos mais restritos. A seguir, ramifica-se a apresentação para estudos mais amplos com intuito de generalizar o conhecimento. Este método de organização destaca como os resultados mais amplos diferem dos mais restritos, e é particularmente útil para revisões orientadas empiricamente.

- Metodológica: agrupa os estudos em função das metodologias empregadas. Uma breve análise contemplando os aspectos cobertos e não cobertos pela metodologia é realizada após a apresentação da mesma. Ao final, uma análise completa compara e resume as conclusões obtidas.

- Por grandes áreas: útil quando existem interpretações diferentes sobre um conjunto de dados. A apresentação pode identificar vários temas e suas diferentes interpretações ou identificar interpretações similares em diferentes áreas.

A RSL desenvolvida por pesquisadores das áreas de educação e psicologia, amplamente adotada por pesquisadores de ciências biomédicas, e que recentemente tem conquistado adeptos em outras áreas da ciência, di- 
fere da RLN por adotar um processo de revisão formal. Explicitado por um conjunto de passos que permitem a verificação e replicabilidade dos procedimentos adotados e das conclusões obtidas pelo pesquisador, contribuindo assim para redução do risco de viés e subjetividade da pesquisa (Tranfield et al., 2003 e De La Torre-UgarteGuanilo et al., 2011).

O processo de execução da RSL pode ser descrito através da sequência de seis passos, conforme sugerido por Soni e Kodali (2011):

- Passo 1: definição do problema de pesquisa de forma clara, objetiva e concisa;

- Passo 2: definição da estratégia de pesquisa, mediante a escolha das bases de dados, do período de pesquisa, dos termos de busca e dos idiomas;

- Passo 3: definição de critérios para seleção de trabalhos que incluem os critérios de inclusão e exclusão;

- Passo 4: seleção dos trabalhos, conforme a estratégia de pesquisa definida no Passo 2 e critérios decididos no Passo 3.

- Passo 5: análise dos trabalhos selecionados, mediante a revisão profunda dos mesmos, considerando apenas as obras selecionadas e relacionadas ao problema de pesquisa definido. Durante esse passo, podem ser empregados métodos complementares para compilar os resultados do estudo, entre os métodos sugeridos estão a Metassíntese e a Meta-análise. $\mathrm{Na}$ Metassíntese, os resultados obtidos na RSL são analisados e compilados de forma qualitativa, buscando obter conclusões macro do conjunto de estudo. Segundo Lopes e Fracolli (2008), a Metassíntese é uma "integração interpretativa de resultados qualitativos que são, em si mesmos, a síntese interpretativa de dados. Tais integrações vão além da soma das partes, uma vez que oferecem uma nova interpretação dos resultados". A Meta-análise é uma técnica utilizada para combinar resultados de diversos trabalhos quantitativos sobre um mesmo tema através de um tratamento estatístico dos dados (Urra Medina e Barria Pailaquilen, 2010). O uso dessa técnica requer conhecimento sobre o tema discutido e sobre o tratamento estatístico empregado para garantir a qualidade dos resultados.

- Passo 6: Neste passo apresenta-se o resultado da análise RSL, que consiste na identificação dos principais autores, periódicos e bases de dados que contribuem para a construção de um referencial teórico de pesquisa sobre um tema; além disso, os resultados alcançados devem permitir ao pesquisador determinar lacunas e as oportunidades de pesquisas futuras. De acordo com Fink (2013), a síntese dos resultados de RSL pode ser descritiva. Sínteses descritivas são interpretações das conclusões da revisão baseadas na experiência dos revisores e na qualidade e conteúdo da literatura disponível.

\section{ANÁLISE DA QUALIDADE NA REVISÃO SISTEMÁTICA DA LITERATURA}

Uma vez apresentados os seis passos de execução do método de RSL, é importante retornar às motivações deste trabalho. Conforme enunciado na seção introdutória, o método de RSL combinado com os métodos de Metassíntese e Meta-análise garantem maior disciplina e transparência na condução da revisão da literatura, reduzindo o viés e a subjetividade das análises, porém essas não são condições suficientes para garantir a qualidade da revisão. Falhas na execução dos passos podem contribuir para resultados inadequados.

$\mathrm{Na}$ literatura, diversos autores sugerem o emprego de processos auxiliares para garantir a qualidade da RSL. Por exemplo, a formulação de uma questão de pesquisa inapropriada ao objetivo proposto ou de uma hipótese de pesquisa não passível de verificação podem conduzir o pesquisador a selecionar trabalhos e fazer inferências pouco relevantes ao problema de pesquisa, quando não equivocadas. Denyer et al. (2008) empregam o processo CIMO (Context, Intervention, Mechanism, and Outcome) estruturado em uma série de perguntas revisáveis para definir a questão de revisão e evitar falhas como as mencionadas anteriormente.

$\mathrm{Na}$ definição da estratégia de pesquisa, uma das falhas mais comuns é o pesquisador ignorar trabalhos da chamada "grey literature", trabalhos disponíveis em formatos pouco acessíveis ou usuais como relatórios de pesquisa, notas de aula, trabalhos ainda não publicados (working papers). Fink (2013) afirma que não se deve ignorar trabalhos que não estão disponíveis em bases eletrônicas de dados, ou ainda não publicados, quando o tópico de pesquisa é novo e está associado a conceitos que ainda não estão incorporados aos títulos de temas oficiais disponíveis em bases eletrônicas. Neste sentido, Daly et. al. (2015) afirmam que o processo de busca na literatura não deve ficar restrito apenas a trabalhos publicados; para os autores, as fontes bibliográficas podem ser, mas não estão limitadas a:

- Vídeos;

- Blogs e post na internet;

- Informações disponíveis em correio eletrônico e outros meios de correspondência de especialistas no tópico;

- Dados e imagens;

- Produtos em processo, incluindo produtos preliminares como os propostos em pesquisas, resumos de pesquisas, relatórios internos e de projetos em andamento; e

- Apresentações e pôsteres de resultados de pesquisa.

Ainda nesta linha de falha, o pesquisador pode ignorar no processo de levantamento das bases de dados e dos periódicos, as que são de difícil acesso, ou selecionar apenas trabalhos nos idiomas de seu domínio, o que pode caracterizar outra fonte de viés na execução da pesquisa.

Ressalta-se nestes dois casos a dificuldade que um pesquisador inexperiente enfrenta. Por exemplo, a decisão sobre a inclusão ou não de trabalhos da "grey literature", um pesquisador inexperiente pode incluir trabalhos de qualidade duvidosa e comprometer os resultados de sua pesquisa. 
Tabela 2: Abordagens de avaliação da qualidade da Revisão Sistemática.

\begin{tabular}{lll}
\hline Autores & Ferramentas de avaliação & Aplicação \\
\hline Popay et. al. (1998) & Questionário de Avaliação de Qualidade & $\begin{array}{l}\text { Restrito à etapa de avaliação da qualidade dos trabalhos } \\
\text { selecionados para revisão. }\end{array}$ \\
\hline Shea et. al. (2007) & $\begin{array}{l}\text { AMSTAR (A MeaSurement Tool to Assess } \\
\text { Reviews })\end{array}$ & Avaliação aplicada ao término da revisão \\
\hline Denyer et al. (2008) & $\begin{array}{l}\text { CIMO (Context, Intervention, } \\
\text { Mechanism, and Outcome) }\end{array}$ & Restrito à etapa de formulação da questão de pesquisa \\
\hline $\begin{array}{l}\text { Denyer e Tranfield } \\
(2009), \text { Fink (2013) }\end{array}$ & Painel de Especialistas & $\begin{array}{l}\text { Nas etapas de formulação da questão de pesquisa, seleção dos } \\
\text { trabalhos e avaliação da qualidade. }\end{array}$ \\
\hline
\end{tabular}

A construção das strings de busca também pode ser uma fonte potencial de falha. A determinação equivocada dos termos de busca como, por exemplo, empregar apenas termos específicos de uma única área ignorando a ocorrência na literatura de sinônimos ou termos similares em outras áreas relevantes limita a abrangência da pesquisa. O emprego inadequado dos operadores lógicos e de truncamento também podem contribuir para surgimento de falhas. Termos de busca equivocados podem conduzir a resultados extremos quanto à quantidade de referências encontradas.

Falhas também podem ocorrer na etapa de seleção dos trabalhos que deverão ser incluídos na RSL. Tranfield $e t$ al. (2003) esclarecem que uma das principais dificuldades do método de RSL é garantir a qualidade da avaliação dos trabalhos feita pelo pesquisador, uma vez que esse processo é subjetivo. Frente a essa dificuldade, pode-se adotar como estratégia de condução da pesquisa a avaliação dos estudos realizada por um painel de pesquisadores.

Outras abordagens que podem ser adotadas são os critérios de qualidade propostos por Popay et al. (1998) ou a ferramenta para avaliação de qualidade das revisões denominada AMSTAR (A MeaSurement Tool to Assess Reviews) proposta por Shea et. al. (2007).

Finalmente, mesmo quando corretamente executado, o método de RSL pode apresentar resultados inadequados quando o pesquisador dedica mais esforços à execução do método do que ao processo de avaliação dos resultados obtidos e de suas implicações para o prosseguimento da pesquisa. Este tipo de falha está vinculado à falta de senioridade do pesquisador em relação ao tema de pesquisa ou ao método de RSL. Esta é uma visão compartilhada por Tranfield et al. (2003), Denyer e Tranfield (2009) e Fink (2013) que afirmam que a RSL deve ser conduzida por um grupo de pesquisadores que possuem senioridade e experiência tanto no tema de pesquisa como na execução do método.

A Tabela 2 resume as diferentes abordagens apontadas na literatura para avaliar a qualidade da RSL.

Em relação às abordagens reunidas na Tabela 2, é importante destacar que algumas delas estão restritas a etapas específicas do RSL, por exemplo, a abordagem CIMO é aplicada apenas à etapa de formulação da questão de pesquisa. Os critérios de Popay et al. (1998) são utilizados para avaliação da qualidade dos trabalhos incluídos na RSL. O painel de especialistas pode ser aplicado às etapas de formulação da questão de pesquisa, de seleção e avaliação da qualidade dos trabalhos, mas exige que ao menos dois pesquisadores com mesmo nível de conhecimento sobre o tema participem da execução da RSL, o que muitas vezes pode representar um desafio prático.

O método AMSTAR é indicado para avaliação da qualidade da RSL após sua condução. Essa forma de aplicação limita a possibilidade de corrigir falhas e aprimorar o processo RSL durante sua condução.

Neste trabalho sugere-se adaptar os critérios de qualidade propostos por Popay et al. (1998) e aplicá-los para avaliar os passos da execução da RSL. O emprego dos critérios de qualidade busca orientar o pesquisador no processo de execução da RSL. Sua aplicação através de um processo interativo proporciona um mecanismo de avaliação e identificação de falhas, que permite ao pesquisador promover as correções necessárias.

\section{MÉTODO}

Para demonstrar a aplicabilidade dos critérios de qualidade na identificação e correção de falhas da RSL foram desenvolvidos três estudos de caso, através do Método de Estudo de Casos Múltiplos de Yin (2005), combinados aos critérios de qualidade adaptados de Popay et al. (1998). O Método de Estudo de Casos Múltiplos de Yin (2005) possui três etapas principais: (i) definição e planejamento; (ii) preparação, coleta e análise dos casos; (iii) análise cruzada dos casos e conclusão.

Na primeira etapa de definição e planejamento foi determinada a pergunta de pesquisa, "Quais falhas podem ser identificadas em uma RSL a partir da aplicação dos critérios de qualidade?". Nesta etapa também é definido o protocolo de pesquisa a ser adotado. Optou-se por utilizar como protocolo os critérios de qualidade adaptados de Popay et al. (1998) apresentados na Tabela 3. Finalmente, foram selecionados três casos de aplicação da RSL conduzidos por pesquisadores de diferente senioridade, formação e graduação acadêmica (mestrado, doutorado e pós-doutorado).

$\mathrm{Na}$ segunda etapa buscou-se identificar as falhas de aplicação do método RSL. Os autores dos trabalhos selecionados foram indagados com base nos critérios de qualidade propostos e as falhas de execução foram identificadas. Neste trabalho, os critérios foram aplicados a posteriori do desenvolvimento da RSL, porém propõe-se que estes critérios sejam aplicados durante a execução da RSL. O processo de avaliação da qualidade deve ser conduzido de forma interativa. $\mathrm{O}$ pesquisador verificará se os critérios são atendidos à medida que os passos da revisão sistemática são desenvolvidos. 
Tabela 3: Critérios de qualidade da pesquisa.

Fonte: Adaptado de Popay et al. (1998)

\begin{tabular}{|c|c|c|}
\hline Aspecto & Questões de avaliação & $\begin{array}{l}\text { Correlação com os } \\
\text { passos da RSL }\end{array}$ \\
\hline Marcador primário & $\begin{array}{l}\text { A pesquisa esclarece o significado subjetivo, as ações e o contexto do objeto } \\
\text { que está sendo pesquisado? }\end{array}$ & Passo 1 \\
\hline $\begin{array}{l}\text { Comprovação de contexto } \\
\text { de resposta e flexibilidade de } \\
\text { projeto de pesquisa }\end{array}$ & $\begin{array}{l}\text { Existem evidências da adaptação ou responsividade do projeto de pesquisa às } \\
\text { circunstâncias e características encontradas durante o curso de execução da } \\
\text { pesquisa? }\end{array}$ & Passo 2 \\
\hline $\begin{array}{l}\text { Comprovação de } \\
\text { amostragem teórica ou } \\
\text { proposital }\end{array}$ & $\begin{array}{l}\text { A amostra selecionada produz o tipo de conhecimento necessário para } \\
\text { entender a estrutura e os processos internos respondendo à questão da } \\
\text { pesquisa? }\end{array}$ & Passos 2 e 3 \\
\hline $\begin{array}{l}\text { Comprovação de descrição } \\
\text { adequada }\end{array}$ & $\begin{array}{l}\text { Nos trabalhos selecionados há uma descrição detalhada suficiente para } \\
\text { permitir ao pesquisador interpretar o significado e o contexto do que está } \\
\text { sendo pesquisado? }\end{array}$ & Passos 4 e 5 \\
\hline $\begin{array}{l}\text { Evidência de qualidade de } \\
\text { dados }\end{array}$ & $\begin{array}{l}\text { Como as diferentes fontes de conhecimento, tanto na amostragem quanto } \\
\text { nos trabalhos selecionados sobre o mesmo assunto, são comparadas e } \\
\text { contrastadas? As percepções são subjetivas? As experiências são tratadas } \\
\text { como conhecimento? }\end{array}$ & Passos $2,3,4$ e 5 \\
\hline $\begin{array}{l}\text { Comprovação da adequação } \\
\text { teórica e conceitual }\end{array}$ & $\begin{array}{l}\text { Como a pesquisa se desenvolve: na descrição dos dados (através da citação ou } \\
\text { exemplos para uma análise e interpretação dos resultados) e, importância da } \\
\text { pesquisa? }\end{array}$ & Passos 5 e 6 \\
\hline $\begin{array}{l}\text { Potencial para avaliar } \\
\text { tipicidade }\end{array}$ & $\begin{array}{l}\text { Quais generalizações podem ser feitas a partir do resultado da pesquisa, quer } \\
\text { seja para outras populações ou grupos ou outras áreas de pesquisa? }\end{array}$ & Passos 5 e 6 \\
\hline $\begin{array}{l}\text { Relevância para a política ou } \\
\text { área de conhecimento }\end{array}$ & $\begin{array}{l}\text { Existe relevância na pesquisa para diferentes "stakeholders". Estes são } \\
\text { indicados? }\end{array}$ & Passos 5 e 6 \\
\hline
\end{tabular}

Por exemplo, o primeiro critério de qualidade busca verificar se os objetivos da revisão são adequados para o pesquisador responder à questão de pesquisa proposta. Caso se verifique que existe discrepância, o pesquisador deverá revisar suas decisões. Neste exemplo, duas ações podem ser adotadas pelo pesquisador: alterar os objetivos ou hipótese da pesquisa ou reformular a questão de pesquisa, para que esta represente de forma clara o objetivo da pesquisa.

Nesse exemplo, é evidente a dificuldade que um pesquisador de menor senioridade experimenta ao aplicar o método de RSL. Apesar do processo de execução estar sistematizado, não existe no método de RSL uma etapa de avaliação ou verificação das decisões tomadas pelo pesquisador quanto à definição da questão de pesquisa, e ou dos termos de busca. Um pesquisador sênior pode, em função de sua experiência, definir com maior precisão se a questão de pesquisa está adequada, se os termos de busca refletem os objetivos da pesquisa e, finalmente, se as bases de dados e periódicos selecionados são relevantes.

Na terceira etapa do Método de Estudo de Caso, os trabalhos selecionados são avaliados de forma cruzada. Esta análise permite identificar se as falhas que ocorreram na execução da RSL são isoladas ou se um padrão pode ser determinado. Após a análise individual e cruzada, algumas considerações sobre as falhas de execução da RSL são discutidas. O fluxograma da Figura 1 apresenta as etapas descritas acima e na próxima seção é apresentada a análise dos casos.

\section{ANÁLISES DOS CASOS}

Foram analisados três artigos científicos desenvolvidos com a metodologia de RSL sobre os temas Logística, Transportes e Cadeia de Suprimentos. O primeiro caso (Santos Jr. et al., 2011) teve como objetivo apresentar uma revisão das abordagens de gestão de riscos em cadeias de suprimentos e avaliar suas aplicabilidades em operações de redes de suprimentos globais. O segundo caso (Santos et al., 2014) buscou ampliar a compreensão sobre a temática Geografia do Tempo (GT) aplicada ao transporte urbano de cargas. Finalmente, o terceiro caso (Noletto, 2014) identificou a participação das embalagens nas atividades logísticas.

A Tabela 4 apresenta os três artigos selecionados através da descrição dos passos executados para a realização da RSL. Nesta tabela podem ser observados os seguintes aspectos dos artigos selecionados: o problema de pesquisa; a estratégia de pesquisa adotada pelos pesquisadores para determinar os termos de busca; o horizonte de tempo a ser pesquisado; as bases de dados selecionadas; os critérios de inclusão e exclusão dos trabalhos; o processo de seleção e avaliação dos mesmos; e as conclusões obtidas.

Percebe-se através dos elementos descritos na Tabela 4 que os três trabalhos selecionados seguiram a estrutura de desenvolvimento da RSL descrita na seção 2. No entanto, eles apresentam diferentes falhas de execução que comprometem em maior e menor grau a qualidade da revisão. 


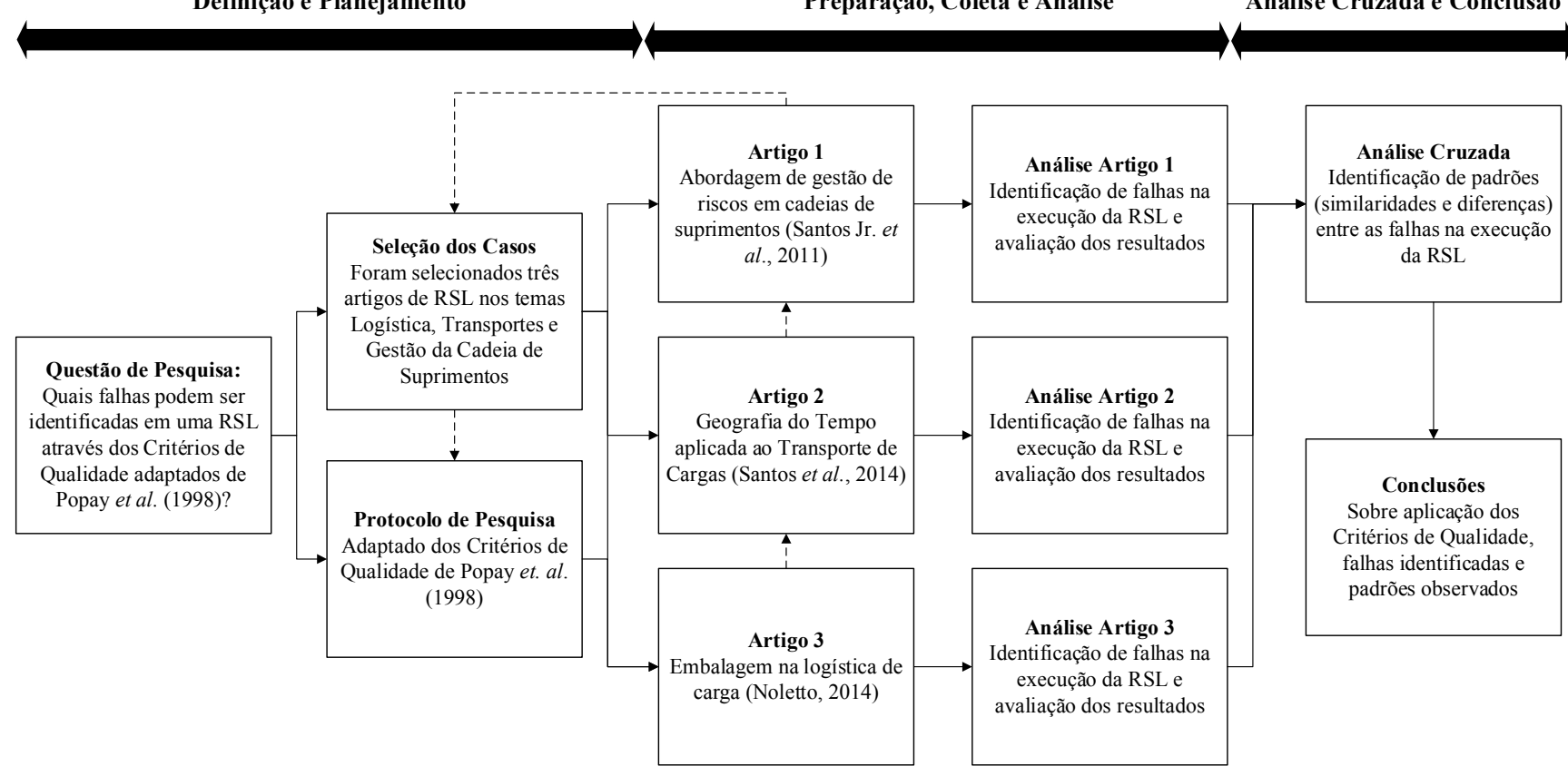

Figura 1: Método de Estudo de Casos Múltiplos. Fonte: Adaptado de Yin (2005).

Os resultados de cada um dos passos conduzidos explicitam as decisões dos pesquisadores. Para avaliar a qualidade destas decisões e a existência de falhas foram realizadas duas análises. A primeira é uma análise individual que busca determinar as falhas de execução da RSL no contexto de cada caso. A segunda análise, denominada cruzada, busca determinar padrões de falhas comuns aos casos. As duas avaliações, individual e cruzada, estão detalhadas respectivamente nas Tabelas 5 e 6 .

A análise dos três estudos de caso nos temas Logística, Transportes e Gestão da Cadeia de Suprimentos, através dos critérios de qualidade propostos, permite identificar falhas na execução da RSL. As falhas identificadas não ocorrem apenas no passo de avaliação da qualidade dos trabalhos selecionados conforme afirmam Tranfield et al. (2003) e De La Torre-Ugarte-Guanilo et al. (2011), mas estão presentes em todos os passos já que todas as decisões tomadas pelos pesquisadores no processo de elaboração da revisão não podem ser consideradas isentas de viés.

$\mathrm{Na}$ Tabela 5, a análise individual dos casos demonstra que falhas de execução nos passos iniciais do método comprometem em maior grau os resultados obtidos. No caso de Noletto (2014), as falhas ocorridas nos passos 1, 2 e 3 interferem nos resultados dos passos 4 e 5 . A questão de pesquisa não claramente delimitada influenciou na escolha equivocada de termos de busca, o que resultou em um encadeamento de erros desde o processo de seleção e escolha dos trabalhos até os resultados obtidos.

Em Santos et al. (2014), a amostra pouco representativa encontrada resulta de falhas no processo de determinação dos termos de busca e/ou da estratégia de seleção das bases e periódicos. Portanto, a análise dos resultados e extrapolação dos mesmos tornam-se limitadas.

Nos três casos estudados, o passo 4 , seleção dos artigos, apresenta elementos insuficientes para replicabilidade da pesquisa. Santos Jr. et al. (2011) explicitam apenas critérios práticos para inclusão ou exclusão de trabalhos, falhando em não apontar os critérios metodológicos utilizados. Falha similar ocorre em Santos et al. (2014) e Noletto (2014).

$\mathrm{Na}$ Tabela 5, também ficam evidenciadas falhas nos passos 5 e 6 , de análise dos trabalhos e conclusões respectivamente. Os três casos seguiram os passos de execução do método de RSL, porém a preocupação com os resultados obtidos e seu potencial para generalização e extrapolação são pouco discutidos.

Santos Jr. et al. (2011) identificam lacunas na literatura sobre gestão de riscos em Cadeia de Suprimentos, porém a discussão realizada é superficial. Não fica claro no artigo como essas lacunas podem ser exploradas; por exemplo, o artigo não explica se as abordagens identificadas são suficientes para endereçar a questão da gestão de risco em cadeias de suprimentos ou se o desenvolvimento de novas técnicas é necessário.

No caso de Santos et. al. (2014), o panorama da Geografia do Tempo aplicada ao transporte urbano de carga é definido através de apenas um trabalho. Neste sentido, o artigo não explora quais os fatores conduzem a um universo tão pequeno de aplicações, como, por exemplo, a existência de limitações nos modelos de Geografia do Tempo, ou inadequações desta abordagem para com os problemas típicos do transporte de carga.

Noletto (2014) apresenta conclusões que permitem identificar linhas de pesquisa e oportunidades de pesquisa futura no tema embalagens em atividades de logística urbana. Porém os resultados do trabalho apresentam viés em função de falhas nos passos iniciais da RSL. Assim, a abrangência dos resultados, a comprovação de amostragem teórica e proposital, a qualidade dos dados e o potencial para avaliar tipicidade são comprometidos.

A Tabela 6 apresenta a análise cruzada dos casos e permite identificar padrões nas falhas encontradas nos três artigos. Três padrões destacam-se nos casos analisados: senioridade do pesquisador, falta de familiaridade com o tema de pesquisa e aplicação do método. 
Tabela 4: Resumo descritivo dos trabalhos selecionados

\begin{tabular}{|c|c|c|c|c|}
\hline \multirow{2}{*}{\multicolumn{2}{|c|}{ Passos da revisão }} & \multicolumn{3}{|l|}{ Casos } \\
\hline & & \multirow{2}{*}{$\begin{array}{l}\text { Santos Jr et al., } 2011 \\
\text { Quais são as principais } \\
\text { abordagens utilizadas para a } \\
\text { gestão de riscos em redes de } \\
\text { suprimentos? }\end{array}$} & \multirow{2}{*}{$\begin{array}{l}\text { Santos et al., } 2014 \\
\text { Qual é o panorama da } \\
\text { literatura internacional } \\
\text { sobre aplicações da GT } \\
\text { ao transporte urbano de } \\
\text { cargas? }\end{array}$} & \multirow{2}{*}{$\begin{array}{l}\text { Noletto, } 2014 \\
\text { Quais são as características } \\
\text { e oportunidades das } \\
\text { embalagens presentes } \\
\text { nas atividades logísticas } \\
\text { urbanas? }\end{array}$} \\
\hline 1. Definição do 1 & roblema de pesquisa & & & \\
\hline \multirow[t]{3}{*}{$\begin{array}{l}\text { 2. Definição da } \\
\text { estratégia de } \\
\text { pesquisa }\end{array}$} & 2.1. Termos de busca & $\begin{array}{l}\text { As combinações } \\
\text { das palavras-chave: } \\
\text { "risk analysis"; "risk } \\
\text { management"; "global } \\
\text { supply chain"; "global } \\
\text { supply network". }\end{array}$ & $\begin{array}{l}\text { As combinações das } \\
\text { palavras-chave: "time } \\
\text { geography"; "space } \\
\text { time geography", } \\
\text { com os termos } \\
\text { "logistics"; "transport"; } \\
\text { "transportation"; } \\
\text { "city logistics"; urban } \\
\text { logistics"; "urban } \\
\text { freight"; "urban cargo"; } \\
\text { "urban logistics". }\end{array}$ & $\begin{array}{l}\text { As combinações das } \\
\text { palavras-chave: "packaging" } \\
\text { com as palavras-chave } \\
\text { "urban logistics", "city } \\
\text { logistics" e "urban freight" }\end{array}$ \\
\hline & 2.2. Horizonte de tempo & De 2000 até 2010. & De 1970 até 2014. & Sem restrição de tempo. \\
\hline & 2.2. Bases de pesquisas & $\begin{array}{l}\text { Emerald; JSTOR; Science } \\
\text { Direct; Taylor \& Francis. }\end{array}$ & $\begin{array}{l}\text { Emerald; Google } \\
\text { Scholar; JSTOR; } \\
\text { Science Direct; Scopus. }\end{array}$ & $\begin{array}{l}\text { Academic Search Premier } \\
\text { - ASP (EBSCO; Emerald; } \\
\text { OneFile (GALE); Science } \\
\text { Direct; Web of Science; } \\
\text { Wiley Online Library. }\end{array}$ \\
\hline \multirow{2}{*}{$\begin{array}{l}\text { 3. Definição } \\
\text { de critérios } \\
\text { para inclusão } \\
\text { ou exclusão de } \\
\text { trabalhos }\end{array}$} & 3.1. Critérios de inclusão & $\begin{array}{l}\text { Artigos que possuam uma } \\
\text { ou mais palavras-chave } \\
\text { definidas no } \\
\text { passo } 3 \text {, considerando o } \\
\text { horizonte de tempo definido } \\
\text { no passo } 2 \text {. }\end{array}$ & $\begin{array}{l}\text { Todos os artigos } \\
\text { científicos sobre } \\
\text { Geografia do Tempo } \\
\text { aplicados ao transporte } \\
\text { (de carga e pessoas). }\end{array}$ & $\begin{array}{l}\text { Artigos que contenham } \\
\text { pelo menos uma das três } \\
\text { combinações de palavras- } \\
\text { chave. }\end{array}$ \\
\hline & 3.2. Critérios de exclusão & $\begin{array}{l}\text { Artigos que não contenham } \\
\text { as palavras-chaves ou fora } \\
\text { do horizonte de tempo. }\end{array}$ & $\begin{array}{l}\text { Todos os trabalhos } \\
\text { que não sejam artigos } \\
\text { científicos. Todos os } \\
\text { resultados repetidos. }\end{array}$ & $\begin{array}{l}\text { Artigos que não estão } \\
\text { escritos em língua inglesa. }\end{array}$ \\
\hline $\begin{array}{l}\text { 4. Seleção } \\
\text { dos artigos }\end{array}$ & & $\begin{array}{l}\text { Foram selecionados } 63 \\
\text { artigos. Foram definidas } \\
\text { cinco categorias (estrutura, } \\
\text { fluxos, finalidade, grau das } \\
\text { relações e da abordagem } \\
\text { técnica.) para classificar } \\
\text { os artigos que tratem a } \\
\text { questão de pesquisa (passo } \\
\text { 1). Finalmente, } 31 \text { artigos } \\
\text { foram selecionados e } \\
\text { analisados. }\end{array}$ & $\begin{array}{l}\text { Identificou-se } 11654 \\
\text { publicações relacionadas } \\
\text { à Geografia do Tempo; } \\
\text { Dessas, selecionou- } \\
\text { se para análise } 284 \\
\text { artigos que tratavam de } \\
\text { Geografia do Tempo } \\
\text { aplicada ao transporte. } \\
\text { Havia apenas } 1 \text { artigo } \\
\text { que atendia totalmente } \\
\text { ao objetivo do trabalho, } \\
\text { ou seja, que possuía } \\
\text { aplicação de Geografia } \\
\text { do Tempo a transporte } \\
\text { urbano de carga. }\end{array}$ & $\begin{array}{l}\text { Após a leitura dos resumos } \\
\text { foram selecionados } 49 \\
\text { artigos. Esses artigos foram } \\
\text { lidos na íntegra obtendo- } \\
\text { se um total de } 36 \text { artigos } \\
\text { voltados para a questão de } \\
\text { pesquisa. }\end{array}$ \\
\hline $\begin{array}{l}\text { 5. Análise } \\
\text { dos artigos } \\
\text { selecionados }\end{array}$ & & $\begin{array}{l}\text { Principais autores e revistas } \\
\text { do tema de pesquisa, as } \\
\text { lacunas identificadas } \\
\text { e oportunidades futuras de } \\
\text { pesquisa }\end{array}$ & $\begin{array}{l}\text { Relação de artigos } \\
\text { publicados por } \\
\text { revista; evolução } \\
\text { das publicaç̃̃es por } \\
\text { ano; evolução das } \\
\text { publicaçôes por } \\
\text { país; análise espacial } \\
\text { abordada; quanto ao } \\
\text { objeto (pessoas, cargas, } \\
\text { informações); e, análise } \\
\text { de estudos que tratem } \\
\text { de transporte urbano de } \\
\text { cargas. }\end{array}$ & $\begin{array}{l}\text { Identificação das principais } \\
\text { revistas que publicam sobre } \\
\text { o tema, distribuição dos } \\
\text { artigos por ano de publicação } \\
\text { e identificação de linhas de } \\
\text { pesquisa inseridas na questão } \\
\text { principal. }\end{array}$ \\
\hline $\begin{array}{l}\text { 6. Apresentação } \\
\text { dos resultados }\end{array}$ & & Artigo científico & Artigo científico & Artigo científico \\
\hline
\end{tabular}


Tabela 5: Análise individual da qualidade da pesquisa

\begin{tabular}{|c|c|c|c|}
\hline \multirow{2}{*}{ Aspecto } & \multicolumn{3}{|l|}{ Análise individual } \\
\hline & Santos Jr. et al. (2011) & Santos et al. (2014) & Noletto (2014) \\
\hline Marcador primário & $\begin{array}{l}\text { Sim. Porque o problema de } \\
\text { pesquisa englobou dois aspectos } \\
\text { principais relacionados à gestão de } \\
\text { riscos em cadeia de suprimentos: } \\
\text { a vulnerabilidade nos processos de } \\
\text { fornecimento; e o impacto de desastres } \\
\text { naturais sobre redes de suprimentos } \\
\text { globais. }\end{array}$ & $\begin{array}{l}\text { Sim. Porque o problema de pesquisa } \\
\text { focou nos dois temas principais } \\
\text { de interesse: geografia do tempo e } \\
\text { transporte de cargas. }\end{array}$ & $\begin{array}{l}\text { O problema de pesquisa não } \\
\text { foi bem delimitado. O escopo } \\
\text { do trabalho ficou amplo, } \\
\text { impossibilitando a formulação } \\
\text { de uma resposta ao problema } \\
\text { proposto. }\end{array}$ \\
\hline $\begin{array}{l}\text { Comprovação de } \\
\text { contexto de resposta } \\
\text { e flexibilidade de } \\
\text { projeto de pesquisa }\end{array}$ & $\begin{array}{l}\text { Sim. A estratégia de pesquisa foi } \\
\text { adequada e a escolha das palavras } \\
\text { atendeu os objetivos. No entanto, o } \\
\text { artigo não informa se houve alterações } \\
\text { nos termos ou strings de busca ao } \\
\text { longo da pesquisa. Caso mudanças } \\
\text { tenham ocorrido, estas não foram } \\
\text { descritas no artigo. }\end{array}$ & $\begin{array}{l}\text { Sim. A estratégia de pesquisa foi } \\
\text { adequada e a escolha das palavras } \\
\text { atendeu aos objetivos. A inclusão } \\
\text { de trabalhos sobre GT aplicada } \\
\text { ao transporte de pessoas difere do } \\
\text { objetivo proposto do trabalho. Porém } \\
\text { o artigo não permite identificar se } \\
\text { houve necessidade de adaptações em } \\
\text { relação aos termos de busca ao longo } \\
\text { do trabalho. }\end{array}$ & $\begin{array}{l}\text { Parcialmente. A estratégia de } \\
\text { pesquisa apresentou problemas. } \\
\text { As palavras chaves escolhidas } \\
\text { não identificaram artigos que } \\
\text { atendessem a pergunta de } \\
\text { pesquisa. Optou-se por ampliar } \\
\text { os termos de busca. O resultado } \\
\text { foi insatisfatório, já que a falha } \\
\text { inicial estava na questão de } \\
\text { pesquisa. }\end{array}$ \\
\hline
\end{tabular}

Sim, a amostra permite identificar gaps e oportunidades de pesquisa no tema gestão de riscos em cadeia de suprimentos. O estudo delimita um

Comprovação de amostragem teórica ou proposital período e contexto do tema pesquisado. Estão claras as strings de busca, as bases de dados analisadas e o protocolo de avaliação, considerando os critérios de seleção, exclusão e avaliação dos trabalhos.

Sim. O pesquisador conseguiu interpretar o significado do contexto do que foi pesquisado através dos

Comprovação de trabalhos selecionados.
A partir da busca realizada foi possível traçar o panorama da literatura sobre o tema de GT aplicado a transportes. A identificação de apenas um trabalho no tema GT em transporte de cargas não permite afirmar que existe comprovação de amostragem teórica ou proposital.

\section{Não. Apesar de identificar} alguns trabalhos relevantes, a estratégia de busca apresentou falhas conforme as questões descritas acima.
Parcialmente, em relação ao panorama da literatura sobre o tema GT. Entretanto, em relação à GT em transporte de cargas não é possível interpretar o significado e contexto do que está sendo pesquisado pois apenas um único artigo foi encontrado.

Identificou um panorama da Geografia do Tempo em diferentes áreas. Se evidencia a possibilidade do desenvolvimento de pesquisas utilizando GT na área de transportes de carga em função dos trabalhos identificados. Mas, o resultado obtido não foi suficiente para traçar um panorama para transporte de cargas.
Não, apesar da pesquisa apresentar um horizonte de tempo, bases de pesquisa variadas e definidos os termos de busca, os trabalhos não conseguiram auxiliar o pesquisador a descrever adequadamente o fenômeno.

Através dos artigos foram identificadas diferentes linhas de pesquisa sobre o tema e as diferentes visões e aspectos sobre o tema foram apresentados e contrastados. No entanto, foram apenas consultados artigos de periódicos em inglês o que restringiu a pesquisa. A pesquisa apresenta um desenvolvimento linear, respeitando as fases de apresentação da questão de pesquisa, o método de investigação a ser empregado, considerando o protocolo de coleta de dados e análise, finalizando com apresentação dos resultados.

O trabalho não permite generalizações, porém traz a colaboração de gerar tipicidades sobre o tema GT aplicado a transporte de cargas a partir de estudos de GT aplicado a transportes de pessoas.

Sim. Uma vez que grande parte dos trabalhos sobre GT aplicados ao transporte de pessoas estão relacionados ao espaço urbano. No entanto, para GT aplicado ao transporte de cargas a relevância da pesquisa para diferentes stakeholders precisa ser avaliada ou melhor identificada.
A pesquisa seguiu todas as etapas descritas no protocolo de RSL. Contudo a amostragem referente ao foco principal da pesquisa foi pequena (apenas 1 artigo) o que limitou a análise proposta inicialmente no trabalho.

Em função do reduzido tamanho da amostra identificada não é possível fazer generalizações com base nos resultados desse artigo.

Sim. Os autores identificam outras áreas e temas inerentes à pesquisa, com diversos públicos interessados.
Relevância para a política relevância para diferentes stakeholders, porém por se tratar de um tema fundamental para gestão da cadeia de gestão de riscos tem potencial para alavancar diferentes empresas envolvidas. 
Tabela 6: Análise cruzada dos artigos selecionados

\begin{tabular}{ll}
\hline Aspecto & Análise cruzada \\
\hline & $\begin{array}{l}\text { Apenas Noletto (2014) não atingiu sucesso no Passo 1 da RSL. Essa falha comprometeu a qualidade da RSL } \\
\text { como um todo, pois influenciou em todas as decisões tomadas nos passos subsequentes. Duas causas podem }\end{array}$ \\
O marcador primário relacionadas a essa falha: a pouca senioridade do pesquisador e/ou seu desconhecimento sobre a área \\
de pesquisa. A definição do problema de pesquisa (Passo 1) deve ser feita por dois ou mais pesquisadores \\
da área de conhecimento e que possuam senioridade de pesquisa suficiente para julgar se a questão proposta \\
encontra-se adequada ao objetivo proposto.
\end{tabular}

Santos Jr. et al. (2011) e Santos et al. (2014) não evidenciaram adaptação ou responsividade do projeto de

Comprovação de contexto de resposta e flexibilidade de projeto de pesquisa pesquisa às circunstâncias e características encontradas durante o curso do projeto. A definição dos termos de busca deve ser feita por dois ou mais pesquisadores da área de conhecimento, de forma a garantir o sucesso na construção do marcador primário. Toda e qualquer adaptação ou responsividade do projeto de pesquisa (caso exista) deve ser evidenciada para facilitar futuras pesquisas na área de estudo. Em Santos et al. (2014), a inclusão de trabalhos no tema Geografia do Tempo aplicada ao transporte de pessoas demonstra uma alteração no projeto de pesquisa não registrada. Essa falha de aplicação indica falta de senioridade dos pesquisadores em possibilitar aos leitores a rastreabilidade da pesquisa que foi executada.

Por possuir um problema de pesquisa ampliado, em Noletto (2014) a amostra não produziu o tipo de conhecimento necessário para entender a estrutura e os processos internos respondendo à questão de

Comprovação de amostragem teórica ou proposital pesquisa. O problema apresentado ocorre como reflexo da falha na realização dos Passos 1 e 2. Em Santos $e t$ al. (2014), a amostra produzida é insuficiente para responder à questão de pesquisa proposta. Os problemas encontrados estão novamente vinculados à questão de falta de senioridade e de experiência na área de aplicação do pesquisador. Estas questões poderiam ser solucionadas através da análise dos resultados obtidos e da revisão dos passos anteriores da RSL.

Por terem realizado os Passos 1, 2 e 3 de forma adequada, Santos Jr. et al. (2011) obtiveram sucesso nos

Comprovação de descrição adequada Passos 4 e 5 referentes à interpretação do contexto do tema da pesquisa. As falhas na execução dos Passos 1, 2, 3 e 4 podem resultar em uma amostra não compatível com os propósitos da pesquisa conforme os resultados obtidos por Santos et al. (2014) e Noletto (2014).

Em todos os casos foi usada apenas uma fonte de conhecimento (artigos oriundos de periódicos), não sendo consideradas outras fontes de pesquisa como teses, dissertações e capítulos de livros, além de outros idiomas, limitando-se à língua inglesa. Entretanto, as percepções não são subjetivas, pois foram embasadas por dados oriundos dos trabalhos selecionados. As experiências não foram tratadas como conhecimento. Uma maior senioridade dos pesquisadores poderia ter estimulado a busca em outras fontes de dados. Essa decisão não indica, necessariamente, um comprometimento da pesquisa realizada, mas deveria estar registrada e justificada no protocolo de pesquisa.

qualidade de dados

Todos os artigos seguiram o protocolo proposto da RSL e seus passos. Santos Jr. et al. (2011) foram bem-sucedidos na execução dos Passos de 1 a 4, proporcionando resultados de acordo com a proposta de pesquisa. Em Santos et al. (2014), a análise foi limitada pela pequena amostragem encontrada a partir dos

Comprovação da adequação teórica e conceitual seus critérios de busca o que comprometeu o resultado esperado. Em Noletto (2014), as falhas nos passos iniciais da RSL limitaram o resultado da proposta de pesquisa. A execução de todas as etapas por si só não garante o sucesso da RSL, portanto, é preciso avaliar de forma crítica os resultados obtidos em cada um dos passos do protocolo de RSL.

Potencial para avaliar tipicidade

Nos três casos, não foi possível realizar generalizações a partir dos resultados das pesquisas. Isso ocorreu devido às restrições de idioma, tipo de material de pesquisa, horizonte de tempo adotadas nas RSL e que impossibilitam generalizações para outros grupos e áreas de pesquisa.

Relevância para a política

Os três estudos têm relevância para diferentes stakeholders, apesar de esta não ter sido claramente identificada em Santos Jr. et al. (2011) e Noletto (2014). Santos et al. (2014) identificam áreas de relevância do tema GT.
As falhas relacionadas à falta de senioridade do pesquisador são evidenciadas nos passos da RSL como, a definição da questão de pesquisa, a determinação de critérios metodológicos de inclusão e exclusão de trabalhos e na análise dos resultados.

A falta de familiaridade do pesquisador com o tema de pesquisa influencia a decisão sobre as fontes de dados que serão pesquisadas, a inclusão ou não de trabalhos de grey literature, além do julgamento sobre a aderência dos resultados encontrados diante das decisões de pesquisa adotadas (questão de pesquisa, critérios de seleção dos trabalhos e base de dados).

As questões referentes à aplicação do método são influenciadas pelos dois padrões de falha descritos acima e podem ser sintetizados na ausência no método de RSL de critérios de avaliação da qualidade que permitem ao pesquisador questionar e verificar suas decisões de pesquisa durante o processo de execução da RSL.

\section{CONCLUSÕES}

O uso do método de Revisão Sistemática da Literatura, embora sistematizado, exige esforço e maturidade de pesquisa incompatível com pesquisas conduzidas por jovens pesquisadores em trabalhos de iniciação científica e mestrado.

A falta de familiaridade do pesquisador com o tema de pesquisa pode penalizar a aplicação do método e os resultados obtidos, principalmente nas etapas iniciais relacionadas à: definição da questão de pesquisa; escolha dos 
termos de busca; e seleção das bases de dados e periódicos a serem consultados.

O grau de exigência e dedicação necessário para a correta aplicação do método de Revisão Sistemática muitas vezes conduz o pesquisador menos experiente a confundirse entre a valorização da aplicação do método e do objetivo da pesquisa propriamente, negligenciando a etapa de conclusão.

A avaliação do método de RSL pode ser organizada segundo três aspectos: método; aplicação do método; e qualidade dos resultados obtidos nas aplicações.

Quanto ao método, a principal qualidade da RSL é permitir a sistematização do processo de busca e avaliação da literatura em determinado tema, direcionando o pesquisador a aprofundar-se na definição de sua pergunta de pesquisa e nas características de seu objeto de estudo. Em contrapartida, como o método é bastante trabalhoso, em vários casos troca-se o objetivo da pesquisa científica em andamento pelo objetivo de se aplicar corretamente o método em todas as suas etapas. Isto ocorre em muitas situações onde a ferramenta passa a ter mais importância que o problema para o pesquisador.

Um aspecto a se destacar é que o método de RSL é uma ferramenta potente para: dar continuidade a pesquisas quantitativas; endereçar problemas com média ou alta complexidade; identificar novos campos de pesquisa, ou áreas em mudança do estágio de maturidade de conhecimento ou que necessitam de organização do referencial teórico, como em pesquisas de doutorado e pós-doutorado. Porém a RSL se apresenta inadequada para problemas de baixa complexidade, com referenciais teóricos consolidados ou já bem explorados na literatura, comumente observado em pesquisas de mestrado e iniciação científica.

A RSL auxilia o pesquisador a construir o referencial teórico que utilizará em sua pesquisa, conduzindo seu estudo a partir dos limites do conhecimento sobre o tema, evitando que o pesquisador repita pesquisas e suas conclusões de forma desnecessária, ou pior ainda, fazendo apuds de qualidade duvidosa.

O desenvolvimento da RSL é facilitado em função da variedade de bases de dados e buscadores disponíveis. No Brasil temos o exemplo do portal periódico CAPES que disponibiliza uma grande quantidade de periódicos nacionais e internacionais, permitindo aos pesquisadores brasileiros acesso à literatura vasta e de qualidade. Assim, uma dificuldade da aplicação do método de RSL não está no acesso, e sim na leitura e elaboração da síntese de um grande conjunto de materiais.

A qualidade dos resultados obtidos está relacionada com a verificação e validação dos passos da RSL e, neste sentido, o artigo propôs o emprego de critérios de qualidade para identificação e correção das falhas à medida que os passos sejam executados. Essa medida pode ser adotada como solução à necessidade de execução do painel de especialistas. O objetivo do painel é aumentar a confiabilidade do processo e reduzir a possibilidade de viés, mas muitas vezes esta medida se mostra de difícil execução.

Finalmente, a RSL demonstra-se insuficiente para os objetivos da pesquisa se o esforço de avaliação e inferência dos resultados são colocados em segundo plano diante o esforço de síntese e tabulação dos resultados. O objetivo da revisão da literatura é determinar as fronteiras do conhecimento em determinado tema e, a partir de seus resultados, o pesquisador deve ser capaz de inferir novas conclusões ou aplicações do conhecimento.

Dessa forma, recomenda-se:

- Avaliar a RSL perante à RLN a respeito do esforço versus o ganho de conhecimento científico. Como critério para esta avaliação, o pesquisador deve observar a complexidade do problema de pesquisa, seu conhecimento sobre o tema, e também o seu grau de senioridade;

- Uma vez selecionada a RSL, os passos executados devem ser avaliados. Sugere-se o emprego dos critérios de qualidade ou o auxílio de pesquisadores de maior senioridade para avaliação das decisões, de forma a garantir a qualidade da pesquisa reduzindo a incidência de falhas e viés na pesquisa;

- A qualidade dos resultados deve ser avaliada, verificando-se a contribuição dos mesmos para responder as questões de pesquisa propostas. Neste aspecto, é importante observar se a revisão: delimita o tema em estudo; permite a identificação de lacunas e oportunidades de pesquisa; e, possibilita vislumbrar a aplicação do conhecimento consolidado em outras áreas, stakeholders e problemas.

\section{AGRADECIMENTOS}

Os autores agradecem ao editor e avaliadores por sua cuidadosa avaliação do manuscrito e seus relevantes comentários e sugestões que contribuíram significativamente para o aprimoramento do texto. Ao Conselho Nacional de Desenvolvimento Científico e Tecnológico (CNPq) pelo apoio ao Projeto Pesquisa Universal 474993/2013-2. A Coordenação de Aperfeiçoamento de Pessoal de Nível Superior (CAPES) pela concessão de bolsas durante o período de realização deste trabalho. Ao Laboratório de Aprendizagem em Logística e Transporte - LALT/UNICAMP pelo auxílio à pesquisa.

\section{REFERÊNCIAS}

Casey, P. \& Landgraf, G. Literature eeviews: how to put it all together. In Avni, A., Burley, P., Casey, P., Cherney, J., Christiansen, L., Daly, J. S., ... \& Yu, H. (2015). Literature searches and literature reviews for transportation research projects. How to Search, Where to Search, and How to Put It All Together: Current Practices. Transportation Research E-Circular (E-C194).

Daly, J. S.; Meier, A.; Winter, K.; Yu, H. Literature searches: how to search. In: Avni, A., Burley, P., Casey, P., Cherney, J., Christiansen, L., Daly, J. S.,\& Yu, H. (2015). Literature searches and literature reviews for transportation research projects. How to Search, Where to Search, and How to Put It All Together: Current Practices. Transportation Research E-Circular (E-C194).

De La-Torre-Ugarte-Guanilo, M. C.; R. F. Takahashi e M.R. Bertolozzi (2011). Revisión sistemática: nociones generales. Revista da Escola de Enfermagem da USP, v. 45, n. 5. 
Denyer, D., Tranfield, D., \& Van Aken, J. E. (2008). Developing design propositions through research synthesis. Organization studies, v. 29, n. 3, p. 393-413. DOI: 10.1177/0170840607088020

Denyer, D., \& Tranfield, D. (2009). Producing a systematic review. In Buchanan, D. (ed.). The Sage Handbook of Organizational Research Methods. London: Sage, p. 671-689.

Fink, A. (2013). Conducting research literature reviews: from the internet to paper. Sage Publications.

Littell, J. H.; J. Corcoran e V. Pillai (2008). Systematic reviews and meta-analysis. New York City, NY: Oxford University Press.

Lopes, A. L. M.; Fracolli, L. A. (2008). Revisão sistemática de literatura e metassíntese qualitativa: considerações sobre sua aplicação na pesquisa em enfermagem. Texto contexto - enfermagem, Florianópolis, v. 17, n. 4, p. 771-778. DOI: $10.1590 / \mathrm{S} 0104-07072008000400020$

Marconi, M. A. e E. M. Lakatos (2010). Fundamentos de metodologia científica. 7. ed. São Paulo: Atlas.

Noletto, A. P. R. (2014). A embalagem no contexto da logística urbana: uma revisão sistemática. Campinas/SP. Laboratório de Aprendizagem em Logística e Transportes - LALT da Universidade Estadual de Campinas - LALT Unicamp. (Artigo não publicado)

Tarchetti, O. P. (2010). Análise comparativa de processos logísticos no setor de serviços. Dissertação (Mestrado) - Universidade Estadual de Campinas, Campinas, São Paulo.

Popay, J.; A. Rogers e G. Williams (1998). Rationale and standards for the systematic review of qualitative literature in health services Research. Qualitative Health Research, v. 8, n. 3, p. 341-351. DOI: 10.1177/104973239800800305

Santos Júnior, J. B. S.; O. F. Lima Júnior; A. G. Novaes e B. Scholz-reiter (2011). A comparative analysis of supply network risk management techniques based on systematic literature review. CONGRESSO DE PESQUISA E ENSINO EM TRANSPORTES, 25, 2011. Anais... Belo Horizonte/MG: ANPET.

Santos, L. S.; S. A. Loureiro; O. F. Lima Júnior; B.V. Bertoncini e O. B. Bezerra (2014). Análise da literatura de geografia do tempo aplicada ao transporte urbano de cargas. CONGRESSO DE PESQUISA E ENSINO EM TRANSPORTES, 28, 2014. Anais... Curitiba/PR: ANPET.

Shea, B. J., Grimshaw, J. M., Wells, G. A., Boers, M., Andersson, N., Hamel, C., ... \& Bouter, L. M. (2007). Development of AMSTAR: a measurement tool to assess the methodological quality of systematic reviews. BMC medical research methodology, v. 7, n. 1. DOI: 10.1186/1471-2288-7-10

Soni, G. e R. Kodali (2011). A critical analysis of supply chain management content in empirical Research. Business Process Management Journal, v. 17, n. 2, p. 238-266. DOI: $10.1108 / 14637151111122338$
Tranfield, D.; D. Denyer e P. Smart (2003). Toward a methodology for developing evidence informed management knowledge by means of systematic review. British Journal of Management, n. 14, p.207-222. DOI: 10.1111/1467-8551.00375

Urra Medina, E e R. M. Barria Pailaquilen (2010). A revisão sistemática e a sua relação com a prática baseada na evidência em saúde. Rev. Latino-Am. Enfermagem, Ribeirão Preto, v. 18, n. 4, Aug.

Van Wee, B., \& Banister, D. (2015). How to Write a Literature Review Paper? Transport Reviews, (ahead-of-print), 1-11. DOI:1 0.1080/01441647.2015.1065456

Yin, R. K. (2005). Estudo de caso: planejamento e métodos. 3. ed. Porto Alegre: Bookman. 